\title{
AN EXPERIMENT TO LOCATE THE SITE IN M87 OF TeV FLARING
}

D. E. HARRIS ${ }^{1}$, M. BEILICKE ${ }^{2}$, C. C. CHEUNG ${ }^{3}$, P. COLIN ${ }^{4}$, D. HORNS ${ }^{5}$, S. LEBOHEC ${ }^{6}$, F. MASSARO ${ }^{7}$, D. MAZIN ${ }^{8}$, R. MUKHERJEE ${ }^{9}$, M. RAUE ${ }^{10}$, L. STAWARZ $^{11}$,

R. WAGNER ${ }^{12}$ and S. WAGNER ${ }^{13}$

1 Harvard - Smithsonian Center for Astrophysics, 60 Garden St., Cambridge, MA 02138, USA; harris@cfa.harvard.edu;

2 Washington University in St.Louis, Campus Box 1105, 1 Brookings Drive, St.Louis, MO 63130,USA, beilicke@physics.wustl.edu;

3 National Research Council Research Associate, resident at Naval Research Laboratory, Washington, DC 20375, USA, Teddy.Cheung.ctr@nrl.navy.mil;

4 Max-Planck-Institut für Physik, D-80805 München, Germany, colin@mppmu.mpg.de;

5 University of Hamburg, Institute for Experimental Physics, Luruper Chaussee 149, D-22761 Hamburg, Germany, horns@mpi-hd.mpg.de;

6 University of Utah, Department of Physics 115 South 1400 East, Salt-Lake-City, UT 84112-0830, USA, lebohec@physics.utah.edu;

7 Harvard - Smithsonian Center for Astrophysics, 60 Garden St., Cambridge, MA 02138, USA, and SLAC National Laboratory and Kavli Institute for Particle Astrophysics and Cosmology, 2575 Sand Hill Road, Menlo Park, CA 94025 fmassaro@stanford.edu;

8 IFAE, Edifici Cn., Campus UAB, E-08193 Bellaterra, Spain, mazin@ifae.es;

9 Barnard College, Physics \&6 Astronomy, New York NY 10027 USA, mukherjee.reshmi@gmail.com;

10 University of Hamburg, Institute for Experimental Physics, Luruper Chaussee 149, D-22761 Hamburg, Germany, martin.raue@desy.de;

11 Institute of Space and Astronautical Science, JAXA, 3-1-1 Yoshinodai, Sagamihara, Kanagawa, 229-8510, Japan and Astronomical Observatory, Jagellonian University, ul. Orla 171,30-244 Kraków, Poland, stawarz@astro.isas.jaxa.jp;

12 Max-Planck-Institut für Physik, D-80805 München, Germany, rwagner@mppmu.mpg.de;

13 Landessternwarte Heidelberg-Koenigstuhl, 69117 Heidelberg Germany, swagner@lsw.uni-heidelberg.de

We describe a Chandra ToO project designed to isolate the site of $\mathrm{TeV}$ flaring in the radio galaxy M87. To date, we have triggered the Chandra observations only once (2010 April) and by the time of our first observation, the $\mathrm{TeV}$ flare had ended. However, we found that the X-ray intensity of the unresolved nucleus was at an elevated level at the time of our first of 9 observations. Of the $\sim 70$ observations we have made of the M87 jet covering 9 years, the nucleus was measured at $>1 \mathrm{keV} / \mathrm{s}$ only 3 times. Two of these occasions can be associated with $\mathrm{TeV}$ flaring, and at the time of the third event, there were no $\mathrm{TeV}$ monitoring activities. We discuss the implications of these data.

Keywords: galaxies: active; galaxies: individual(M87); galaxies: jets; X-rays: general 


\section{The Problem}

Although M87 is normally a weak source at TeV energies, occasionally there is flaring activity which lasts for some days. During these times, the observed TeV intensity can peak at $\geq 10 \%$ Crab. To date there have been 3 well documented flarings:

- 2005 April - H.E.S.S. found highly variable TeV emission with typical timescales of a few days ${ }^{1}$. Since this event coincided with the peak of the giant flare (radio/uv/X-ray) from the knot HST-1 which lies at $0.86^{\prime \prime}$ (60pc in projection) from the nucleus of M87, there has been an on-going debate as to whether the $\mathrm{TeV}$ emission originated near the SMBH or from HST-1.

- 2008 Feb - A second flaring event was observed by several Cherenkov observatories. By chance, a series of VLBA observations was underway by R. C. Walker at $43 \mathrm{GHz}$ : the milli arcsec nucleus progressivly brightened ${ }^{2}$.

- 2010 Apr - This event was observed by H.E.S.S., MAGIC, and VERITAS ${ }^{3}$. Once the reality of the flaring was established, a Chandra ToO was triggered and these results are the subject of this presentation. Although HST-1 has declined from its peak intensity in 2005 to levels similar to those in 2000, the nuclear X-ray emission was at a somewhat higher level than usual. However, no activity at $43 \mathrm{GHz}$ was detected from the nucleus. For more details, see the contribution by Raue at this meeting.

From the $\mathrm{TeV}$ variability time scale, it is deduced that the $\mathrm{TeV}$ emitting region is of the order of a few light days times $\delta$, the unknown beaming factor. Although we have not found such short time scale variability in the X-rays from HST-1, the nucleus has a somewhat shorter time scale for variability than the 20 days of $\operatorname{HST}-1^{6}$.

So the open question is, can we determine the site of the $\mathrm{TeV}$ flaring by identifying common features in the X-ray lightcurves of any of the labelled features in fig. 1 with the $\mathrm{TeV}$ lightcurve?

\section{The Experiment}

The basic idea for the Chandra ToO is to trigger a series of observations when the TeV exhibited a level of $7 \%$ Crab. With luck, we had hoped that the X-ray light curve of either the nucleus or HST-1 would divulge some feature that would correspond to the $\mathrm{TeV}$ excursions. The main problem for this experiment is the brevity of the $\mathrm{TeV}$ flaring compared to the time it takes for mission planning to alter the observing schedule and upload a revised observing schedule.

Our strategy was to make the first observation as soon as possible, and then to follow the first with 4 more $5 \mathrm{ks}$ observations spaced at intervals of between 1.5 and 3 days. We then had 4 more observations, the first of which would start at the beginning of the next dark of the moon fortnight, and these would be spaced by $3 \pm 1$ days. 


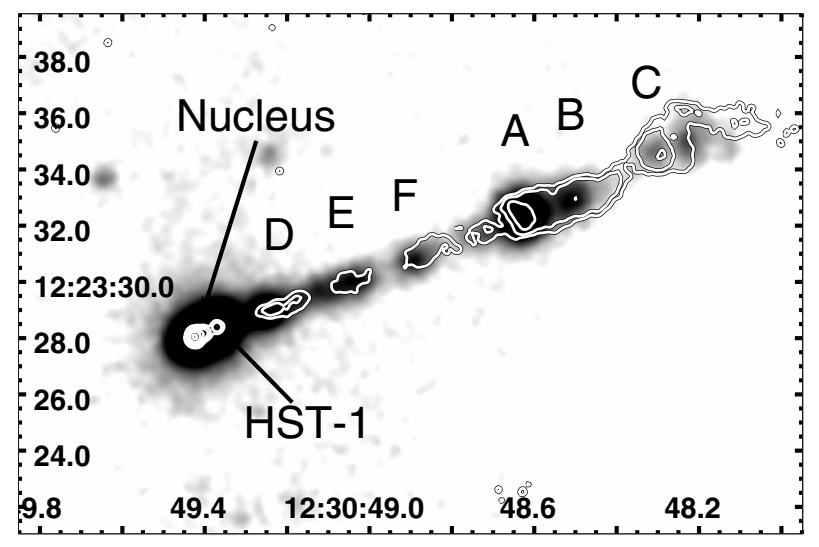

Fig. 1. A Chandra X-ray image of the M87 jet with $8 \mathrm{GHz}$ radio contours overlaid.
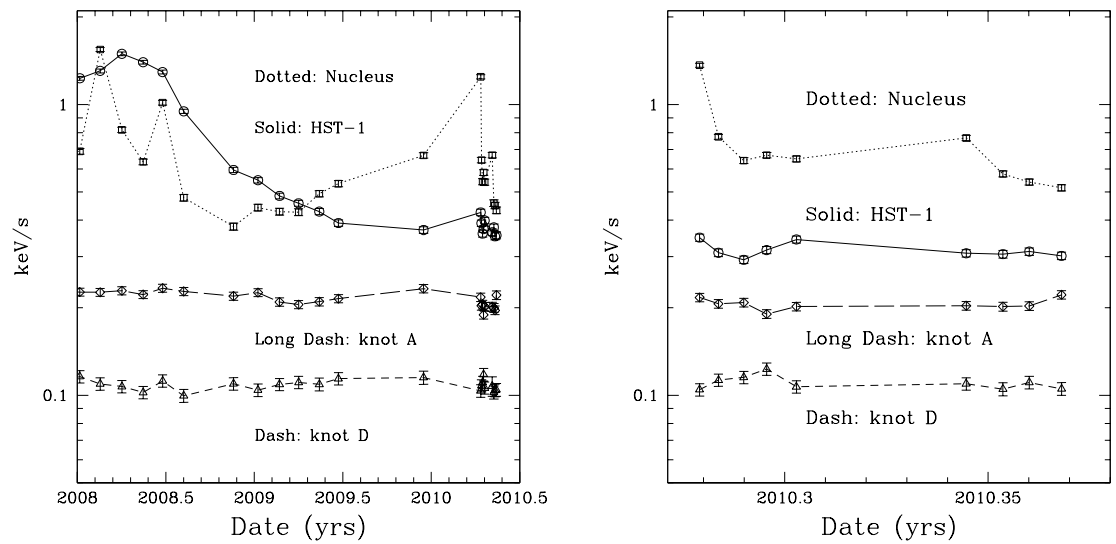

Fig. 2. The X-ray lightcurves for the nucleus, HST-1, knot D, and knot A. left The X-ray lightcurves for two years preceeding the 2010 Apr flaring. right The 9 observations of this experiment. The y axis corresponds to 9 April; our first observation was on 11 April. Shortly before our first observation, there were no more $\mathrm{TeV}$ detections.

On Friday, 9 April 2010 the trigger level was realized and our first observation was late in the day on Sunday, 11 April. Unfortunately, the final elevated TeV level occurred on Saturday night; by Sunday night when we observed, the TeV emission was too low for detection.

\section{The Results}

If the predominant emission mechanism for the nuclear X-rays is synchrotron emission as it is for HST-1 $1^{4,5,6}$, then there must be a sizable population of high energy 
relativistic electrons whose IC emission will be in the TeV range. In fig. 2 we show the light curves for the nucleus and knots HST-1, D, and A. In the right panel, note the high level of the nucleus at our first observation and the sharp drop to more normal levels by the time of the second observation. For comparison, the left panel of fig. 2 shows the same sort of lightcurves for the preceeding 2 years.

In one sense, our experiment failed, but in another, it yielded tantalizing hints that we most likely witnessed the tail end of a period of X-ray flaring associated with the TeV flaring. The intriging question is what we might have seen if Chandra observations had begun a few days earlier. If the slope of the nuclear light curve before our first observation was similar to that between the first two observations, the X-ray intensity of the nucleus during the TeV flaring would have been remarkable.

During our 8 years of monitoring the M87 jet with Chandra, the nuclear emission has seldom been larger than $1 \mathrm{keV} / \mathrm{s}$ : on two occasions (2008 Feb 16 and $2010 \mathrm{Apr}$ 11) these levels were in close proximity to TeV flaring. A third time (2006 Jun 28), there was no TeV coverage, and for the 2005 April TeV flaring, our data are not reliable because of second order pileup affects associated with HST-1. A more detailed analysis of these data, including evidence for a spectral dependency on the rate at which the nuclear intensity dropped between our first two observations, is scheduled to appear in the 10 Dec 2011 issue of the ApJ ${ }^{7}$.

Acknowledgments The work at SAO was supported by NASA grant GO011120X. FM acknowledges the Fondazione Angelo Della Riccia for the grant awarded him to support his research at SAO during 2011 and the Foundation BLANCEFLOR Boncompagni-Ludovisi, n'ee Bildt for the grant awarded him in 2010 to support his research.

\section{References}

1. F. A. Aharonian et al., Science 314, 1424 (2006).

2. V. A. Acciari et al., Science 325, 444 (2009).

3. R. A. Ong et al., Astron. Telegram \#2542, (2010).

4. D. E. Harris et al., Astrophys. J. Lett. 586, L41 (2003).

5. D. E. Harris et al., Astrophys. J. 640, 211 (2006).

6. D. E. Harris et al., Astrophys. J. 699, 305 (2009).

7. D. E. Harris et al., Astrophys. J. 743 (2011). 